\title{
Privacy-preserving Decentralized Federated Deep Learning
}

\author{
Xudong $\mathrm{Zhu}^{*}$ \\ zhudongxu@vip.sina.com \\ Xi'an University of Architecture and Technology (XAUAT) \\ Xi'an, Shaanxi, China
}

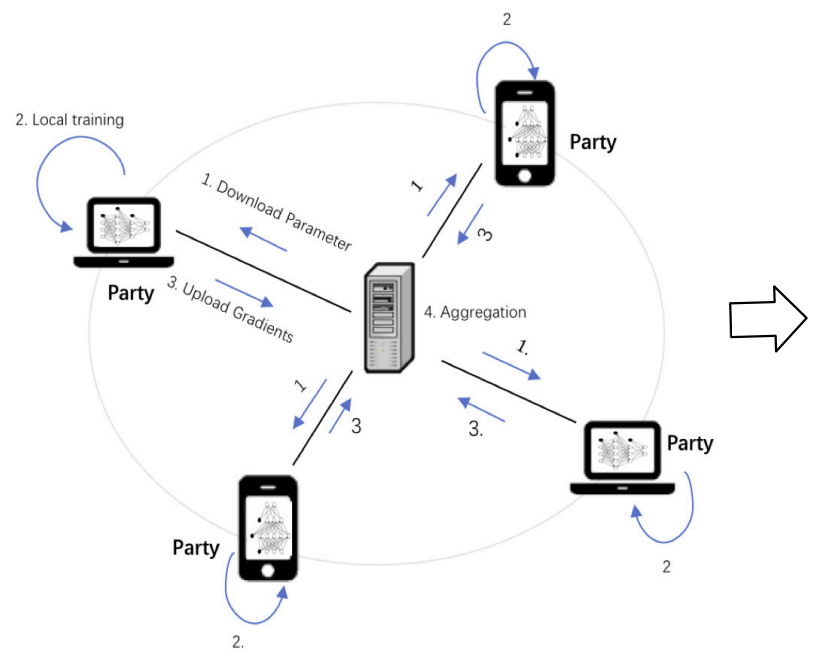

a) FDL
Hui Li

lihui@mail.xidian.edu.cn

Xidian University

Xi'an, Shaanxi, China

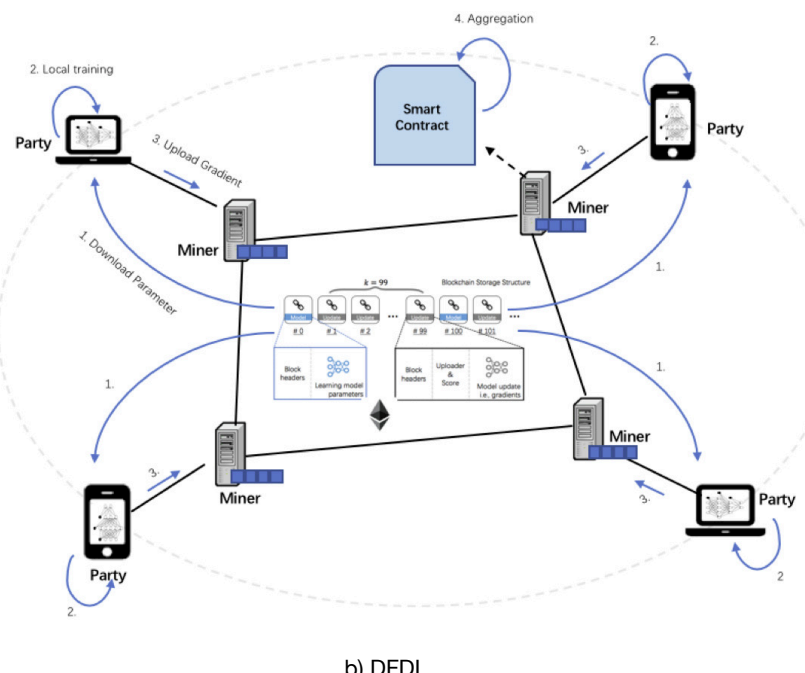

Figure 1: Architectures of Federated Deep Learning (FDL) and Decentralized Federated Deep Learning (DFDL).

\begin{abstract}
Deep learning has achieved the high-accuracy of state-of-the-art algorithms in long-standing AI tasks. Due to the obvious privacy issues of deep learning, Google proposes Federal Deep Learning (FDL), in which distributed participants only upload local gradients and and a centralized server updates parameters based on the collected gradients. But few users are willing to participate in federated learning due to the lack of contribution evaluation and reward mechanisms. So a decentralized federated deep learning, called DFDL, has been proposed by introducing blockchain to form an effective incentive mechanism for participants. However, DFDL still faces serious privacy issues as blockchain does not guarantee the privacy of training data and model. In this paper, in order to address the aforementioned issues, we propose a new Privacy-preserving DFDL scheme, called PDFDL. With PDFDL, parties can securely learn a global model with their local gradients in the assistance of blockchain, and the parties' sensitive data and the global model are

Permission to make digital or hard copies of all or part of this work for personal or classroom use is granted without fee provided that copies are not made or distributed for profit or commercial advantage and that copies bear this notice and the full citation on the first page. Copyrights for components of this work owned by others than ACM must be honored. Abstracting with credit is permitted. To copy otherwise, or republish, to post on servers or to redistribute to lists, requires prior specific permission and/or a fee. Request permissions from permissions@acm.org.

ACM TURC, fuly 30-August 1, 2021, Hefei, China

(C) 2021 Association for Computing Machinery.

ACM ISBN 978-1-4503-8567-1/21/07 . \$ \$15.00

https://doi.org/10.1145/3472634.3472642
\end{abstract}

well protected. Specifically, with a secure multi-party aggregation computing, all local gradients are encrypted by their owners before being sent to the smart contract, and can be directly aggregated without decryption. Detailed security analysis shows that PDFDL can resist various known security threats. Moreover, we give an implementation prototype by integrating deep learning module with a Blockchain development platform (Ethereum V1.6.4). We demonstrate the encryption performance and the training accuracy of our PDFDL on benchmark datasets.

\section{CCS CONCEPTS}

- Security and privacy $\rightarrow$ Privacy protections.

\section{KEYWORDS}

federated deep learning, privacy-preserving, blockchain

\section{ACM Reference Format:}

Xudong Zhu and Hui Li. 2021. Privacy-preserving Decentralized Federated Deep Learning. In ACM Turing Award Celebration Conference - China ( ACM TURC 2021) (ACM TURC), fuly 30-August 1, 2021, Hefei, China. ACM, New York, NY, USA, 6 pages. https://doi.org/10.1145/3472634.3472642

\section{INTRODUCTION}

Recent advances in deep learning methods have led to breakthroughs in long-standing AI tasks such as speech recognition [6], image recognition [2], drug discovery [4] and gene analysis for cancer research [10], [3]. While the utility of deep learning is undeniable, the 
same training data that has made it so successful also presents serious privacy issues [1] [18] [16]. To address such privacy challenges, McMahan et al. [13] proposed a collaboratively distributed deep learning paradigm for mobile devices called Federated Deep Learning (FDL). As shown in Fig. 1 (a), FDL is essentially the combination of deep learning and distributed computation, where there are a parameter server maintaining a deep learning model to train and multiple parties that take part in the distributed training process. First, the central parameter server presents a pre-trained global model on a public dataset to each party. Then, each party trains and improves the current global model on the local dataset in each iteration. Upon receipt of the gradients from all the parties, the central parameter server aggregates those gradients to generate a new global model for the next iteration. Finally, both the parties and the central parameter server repeats the above process until the global model reaches a certain accuracy or optimal convergence.

Although FDL has shown some effectiveness in protecting privacy, it still faces many challenges. First, a single central server is prone to a single point of failure, and can skew the global model by favoring some parties. Moreover, some malicious central servers can poison the model and even collect parties' privacy data from updates. Then, the centralized FDL framework is operated by some voluntary parties and there lacks an efficient incentive mechanism to motivate the participation of parties [11]. To solve its limitations mentioned above, Decentralized Federated Deep Learning (DFDL) has been proposed [8] [5] [15] [19]. As shown in Fig. 1 (b), DFDL consists of task publisher, miner and parties, wherein a learning task is first published by a task publisher, and then completed by the cooperation of several training parties and miners. While blockchain is introduced to track each FDL-related event, such as publishing a task, broadcasting learning models, and aggregating learning results. And a smart contract (SC) is used to replace the central server, verify the uploaded parameters, and provide rewards proportional to the training sample sizes.

However, despite the introduction of blockchain, DFDL still faces a number of data security and privacy challenges. Firstly, DFDL cannot protect the privacy of training data and model parameters, even all intermediate gradients and model parameters have been already stored in the blockchain. For example, intermediate gradients that anyone can obtain from the blockchain at any time can be used to infer important information about training data [17], [14]. To avoid this problem, Liu et al. [12] uploaded the noise-added gradient onto the blockchain to achieve a balance between data privacy and training accuracy. But Liu's work has been pointed out that cannot protect data privacy, since any curious participant can download the gradients from the blockchain and then learn privacy data through Generative Adversarial Network (GAN) learning [7]. Secondly, the malicious attack of parties can disrupt the collaborative training process and poison the DFDL model. For example, malicious parties may submit error gradients deliberately. Therefore, these issues compel the need to develop a secure and privacy-preserving framework for DFDL, so that more parties can actively involved in collaborating training.

In the paper, we propose a privacy-preserving decentralized federated deep learning scheme, named PDFDL. In PDFDL scheme, smart contracts (SC) can securely aggregate local intermediate gradients and update model parameters from untrusted parties through launching transactions. Meanwhile, we use multi-party secure computing technology to ensure the gradients and parameters confidentiality. To summarize, in this paper we made the following contributions:

- First, PDFDL addresses the privacy and data security issues of decentralized federated deep model learning. With PDFDL, the private local model gradients of parties are encrypted with a modified paillier crypto-system, and are operated without decryption. Therefore, the sensitive training data of parties can be well protected, meanwhile, the confidentiality of the final global model is ensured.

- Second, PDFDL achieves decentralized federated deep model learning accurately. To achieve this goal, we construct a secure multi-party aggregation algorithm based on cryptosystem with threshold decryption, which supports lossless collaborative model learning while protecting the data privacy of participants.

- Third, we implement a prototype of PDFDL and evaluate it in terms of ciphertext size, throughput, training accuracy and training time.

The remainder of this paper is organized as follows. In section 2 , we formalize the system model, the threat model and security requirements. Then we propose our PDFDL in section 3, followed by the security analysis and performance evaluation in section 4 and section 5, respectively. Finally, we draw our conclusions in section 8 .

\section{MODEL AND SECURITY REQUIREMENT}

In this section, we formalize the system model and security requirements.

\subsection{System Model}

In our system model, we mainly focus on how to provide privacypreserving decentralized federated deep learning. The system consists of four parts: 1) Task Publisher (TP), 2) Parties (P), 3) Miners (M) and 4) Smart Contract (SC). TP and $\mathbf{P}$ are connected to its local miners, which forms a blockchain network. SC, which is deployed on the blockchain by TP, can aggregate the local gradients from parties and update the global model parameters. As shown in Fig.

- TP is a task publisher which broadcasts a federated learning task through deploying a smart contract over the blockchain network. In the SC, TP need to save rewards as financial incentives for learning tasks, and generates and distributes the initial model parameters and distributing keys for parties.

- $\mathbf{P}=\left\{P_{1}, P_{2}, \cdots, P_{n}\right\}$ is a set of $n$ parties. Each $P_{i} \in \mathbf{P}$ owns a set of data samples $S_{i}$ with $\left|S_{i}\right|=N_{i}$, downloads the current global model from its associated miner, trains its local model, and broadcasts encrypted local model updates $C_{P_{i}}\left(\triangle \mathbf{W}_{i}^{t}\right)$ to its associated miner via transaction $\operatorname{Tran}_{P_{i}}^{t}$, where $C$ is a cipher used by $P_{i}$ to encrypt the weight gradients $\Delta \mathbf{W}_{i}^{t}$.

- $\mathbf{M}=\left\{M_{1}, M_{2}, \cdots, M_{m}\right\}$ is uniformly randomly selected out of a set of $m$ miners. After receiving local updates from all parties before a preset timestamp, each miner updates the global model according to the aggregation rules defined in 


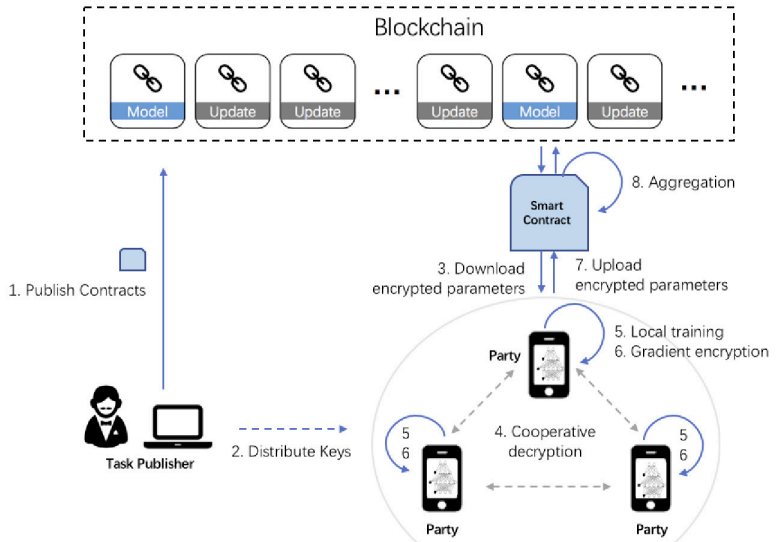

Figure 2: System model under considered.

the SC. Then, by a consensus protocol, the first winning miner generates a new block and other miners verify the contents of this block. Finally, the block which is verified by the majority of miners is added on the blockchain and accepted by the whole network.

- SC is the smart contract which is deployed on the blockchain using the consensus mechanism. SC implements the functions of the centralized parameter server and is actuated by transactions on the blockchain. Specifically, SC computes $C\left(\mathbf{W}_{t+1}\right)=\frac{1}{N} \cdot C\left(\mathbf{W}_{t}\right) \cdot \prod_{i=1}^{N} C_{P_{i}}\left(\Delta W_{i}^{t}\right)$ where $C\left(\mathbf{W}_{t}\right)$ is the encrypted weight at round $t$ in $\operatorname{Tran}_{c o}^{i}$ and updates parties' local models and $C\left(\mathbf{W}_{t+1}\right)$ is the encrypted update weights that will be attached to $\operatorname{Tran}_{c o}^{i+1}$ for updating the local models in next round $i+1$. Finally, SC rewards the miners as well as the training parties according to their contributions in the learning task.

\subsection{Threaten and Security Requirement}

In our threaten model, we consider that parties and miners are a weaker threat model than honest-but-curious. Specifically, 1) malicious parties can inspect all messages received from blockchain including the model iterates in the rounds and can tamper with the training process. 2) malicious miners can inspect all messages sent to blockchain including the gradient updates in all rounds and can tamper with the training process. 3) malicious parties can have a white-box access to the learned model. Considering above security issues, the following security requirements should be satisfied.

- Privacy. Protecting the privacy of each party's local training data. Concretely, during the federated deep learning process, every party's local training data cannot be leaked to anyone.

- Confidentiality. Protecting the global model parameters from blockchain. Specifically, after the federated deep learning process, the global model can only be retrieved by parties.

\section{PROPOSED PRIVACY-PRESERVING SCHEME}

In this section, we present our PDFDL scheme, which mainly consists of four phases: 1) setup; 2) training; 3) aggregation; 4)update. The overview of PDFDL is described in Fig. 3. At first, TP generates system parameters, calculates the public key $P K$ and corresponding private key $S K$ of paillier crypto-system, and splits the private key $S K$ into multiple parts for parties to achieve threshold decryption. Then, parties perform local model training and encrypt their local gradients, which will be submitted to blockchain later. Miner executes cooperative computing with parties to obtain the federated learned result, and send the result to blockchain. Finally, parties can obtain the global model parameters via cooperative decrypting the learned result.

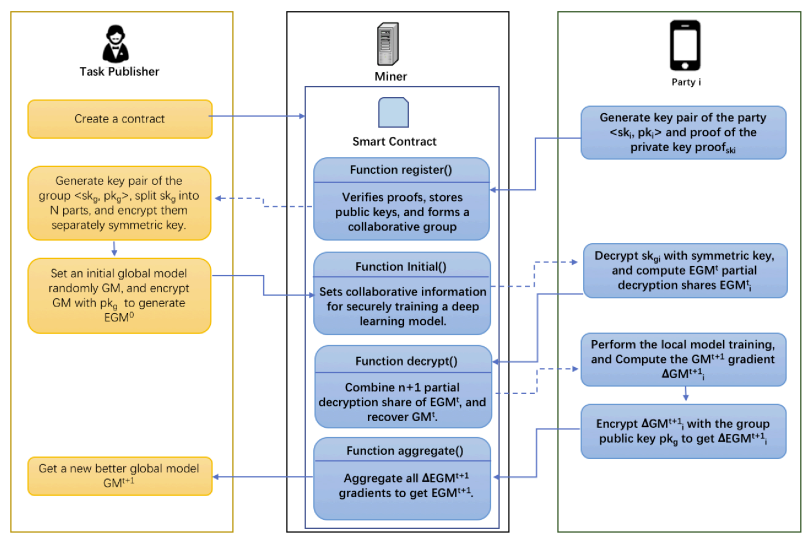

Figure 3: Overview of PDFDL.

\subsection{Setup Phase}

Step 1: Bootstrapping. The Ethereum Blockchain bootstrapping consists of two steps, i.e., ETH tokens distribution and genesis block generation. Assume that all devices and miners have a valid account in the blockchain, where each one uses an address $p k$ that corresponds to a ETH token for launching a transaction. Firstly, ETH tokens distribution realizes ETH allocation among devices and miners, and initially each device or miner is allocated with the same amount of ETH. Then a genesis block is generated at round 0, which contains initial transactions recording ownership statements for each ETH token.

Step 2: Deployment. A task publisher TP, who can be any device that tries to improve the accuracy of its model, broadcasts a federated learning task through deploying a smart contract over the Ethereum blockchain network. The deployed smart contract includes an initial global model, evaluation criteria, and a reward amount. Where the evaluation criterion is the accuracy of federated deep learning model. The reward is a monetary reward, such as "ETH" tokens. The reward is a monetary reward, such as "ETH" tokens, as financial incentives to the learning task.

Step 3: Registration. Each participating device $P_{i}$ prepares a keypair $<s k_{i}, p k_{i}=g^{s k_{i}}>$ and sends the public key $p k_{i}$ together with a non-interactive zero-knowledge proof $\operatorname{proof}_{s k_{i}}$ of 
knowledge of the secret key $s k_{i}$ [1] to the smart contract. Where $\operatorname{proof}_{s k_{i}}$ is the pair $(c, r)$, and $v \in_{R} \mathbb{Z}_{q}, t=g^{v}, c=H\left(g, p k_{i}, t\right)$, and $r=\left(v-c \cdot s k_{i}\right)(\bmod q)$ are called commitment, challenge, and response, respectively. The proof $\operatorname{proof}_{s k_{i}}$ is used to prevent a rogue key attack [2], in which an adversary crafts a public key in an aggregate manner, thereby canceling out the keys from other participants. In addition, the proof is used to tie the participant's Ethereum account to the submitted public key, preventing an adversary from registering itself with a public key copied from an honest participant. The smart contract verifies the proof $\operatorname{proof}_{s k_{i}}$, i.e. $c \stackrel{?}{=} H\left(g, p k_{i}, g^{r} p k_{i}^{c}\right)$, stores the public key received, assigns the registrant an incrementing $i d \in\{1,2, \ldots, n\}$ and triggers a Registration event to notify all participants.

Step 4: Initialization. After the collaborative group is formed, the task publisher TP chooses a security parameter $\alpha$ and two large safe prime numbers $p, q$, where $|p|=|q|=\alpha$, and computes $N=p q$, $\lambda=\operatorname{lcm}(p-1, q-1)$. Then, the task publisher TP chooses a generator $g$ order $(p-1)(q-1) / 2$, such as $g=-a^{2 N}$, where $a$ is a random number in $\mathbb{Z}_{N^{2}}^{*}$. The public key is $P K=N$, and the corresponding private key is $S K=\lambda$. Furthermore, the task publisher set a threshold $u$, s.t., $u-1 \leq n$, and picks a secret polynomial $f: \mathbb{Z}_{N^{2}} \rightarrow \mathbb{Z}_{N^{2}}$ with coefficients $a_{0}=\lambda$ and $a_{1}, a_{2}, \cdots, a_{u-1}$ drawn uniformly at random from $\mathbb{Z}_{N^{2}}$ :

$$
f(x)=a_{0}+a_{1} x+a_{2} x^{2}+\cdots+a_{u} x^{u}\left(\bmod N^{2}\right)
$$

Then, the task publisher TP can split the private key $S K$ into $n$ parts through computing $s_{i}=f(i)$ for all parties $P_{i} \in \mathbf{P}$ and compute $\Delta=n !, v \in \mathbb{Z}_{N^{2}}^{*},\left\{v_{i}=v^{\Delta s_{i}}\right\}_{i \in[1, \ldots, n]}$ which are public verification information where $v_{i}$ corresponds to $s_{i}$. To securely send shares $s_{i}$ to all participants $P_{i} \in \mathrm{P}$, the task publisher TP use a symmetric key encryption algorithm $E n c_{k_{T P, i}}$ ensure secrecy of a sent share from TP to $P_{i}$. The corresponding encryption key $k_{T P, i}$ can be derived non-interactively by both parties:

$$
k_{T P, i}=p k_{i}^{s k_{T P}}=p k_{T P}^{s k_{i}}=g^{s k_{i} s k_{T P}}
$$

Then, the task publisher $T P$ send a transaction $\operatorname{Tran}_{T P}^{\text {init }}$ to the smart contract. Specifically, $\operatorname{Tran}_{T P}^{\text {init }}$ is in the following form.

$$
\operatorname{Tran}_{T P}^{\text {init }}=\left\{P K, \text { commit }_{S K}, \mathbf{V}=\left(v,\left\{v_{i}\right\}_{i \in[1, \ldots, u]}\right), C\left(\mathbf{W}_{0}\right)\right\}
$$

Where $P K$ is the public key, commitment commit $_{S K}$ is obtained by combining parties' commitments on their secret shares $s_{i}$, i.e.

$$
\begin{gathered}
\text { commit }_{S K}=\left(\overline{s_{1}}=E_{n c_{k_{j 1}}}\left(s_{1}\right), \overline{s_{2}}=E n c_{k_{j 2}}\left(s_{2}\right), \cdots,\right. \\
\left.\overline{s_{n}}=E n c_{k_{j n}}\left(s_{n}\right)\right)
\end{gathered}
$$

, and $C\left(\mathbf{W}_{0}\right)$ is the encrypted initial weights of the collaborative model model $_{c o}$, i.e.,

$$
C\left(\mathbf{W}_{0}\right)=g^{\mathbf{W}_{0}} \cdot\left(k_{0}\right)^{N}
$$

Finally, the smart contract stores $\operatorname{Tran}_{T P}^{\text {init }}$ and triggers a Initialization event to notify all parties.

\subsection{Training Phase}

Step 1: Decryption. Suppose that the latest epoch is $t$, the cipher of the latest model weights is $C\left(\mathbf{W}_{t}\right)$ from blockchain and we denote it as $C_{t}$ for brevity. Firstly, each party $P_{i}$ downloads $C_{t}$ and decrypts its share to obtain $s_{i}=\operatorname{Dec}_{k_{i j}}\left(\overline{s_{i}}\right)$. Then, $P_{i}$ generates the partial decryption share $C_{t i}=C_{t}^{2 \Delta s_{i}} \bmod N^{2}$ by using its secret share $s_{i}$. And $P_{i}$ provides the corresponding proofs $\operatorname{Proo} f_{C D_{t, i}}$ for correct shares $C_{t i}$, i.e.,

$$
\operatorname{Proof}_{C D_{t, i}}=\operatorname{fsprove}_{C D}\left(\Sigma_{C D} ;\left(C_{t}, C_{t i}, v, v_{i}\right) ; \Delta s_{i} ; p k_{P_{i}}\right)
$$

Then $P_{i}$ send a transaction $\operatorname{Tran}_{P_{i}}^{D e c}=\left\{C_{t i}\right.$, $\left.\operatorname{Proof}_{C D_{t, i}}\right\}$ to the smart contract. Finally, the smart contract verifies validity of the decryption shares, i.e., $\Delta s_{i}=\log _{C_{t}^{4 \Delta}}\left(C_{t, i}^{2}\right)=\log _{v^{\Delta}}\left(v_{i}\right)$, through

$$
\operatorname{fsver}_{C D}\left(\Sigma_{C D} ;\left(C_{t}, C_{t, i}, v, v_{i}\right) ; \operatorname{Proof}_{C D_{t, i}} ; p k_{P_{i}}\right)
$$

And by combining $t+1$ valid partial decryption shares of the subset $S$ of the parties, the smart contract can correctly recover to plaintext $\mathrm{W}_{t}$ by

$$
\mathbf{W}_{t}=\frac{\prod_{i \in S} C_{t, i}^{2 \mu_{i}}-1}{N} \times \frac{1}{4 \Delta^{2} \theta} \bmod N
$$

where $\mu_{i}$ is the Lagrange interpolation coefficient with respect to $P_{i}$. And the smart contract triggers a Decryption event to push $\mathbf{W}_{t}$ to all parties.

Step 2: Local Model Training. Each parties $P_{i}$ runs stochastic gradient descent (SGD) on the local dataset and updates the model parameters $\mathbf{W}_{t+1}^{i}$ according to

$$
\mathbf{W}_{t+1}^{i}:=\mathbf{W}_{t}-\alpha \frac{\partial \mathbf{E}_{t}}{\partial \mathbf{W}_{t}}
$$

where $\alpha$ is the learning rate and $E_{t}$ is the error function i.e., the difference between the true value of the objective function and the computed output of the network. And each party $P_{i}$ computes gradient vector $\Delta \mathbf{W}_{t}^{i}$ which is the vector of changes in all local parameters due to SGD.

Step 3: Gradient Encryption. In order to achieve the privacy of local model gradients, every device $P_{i}$ encrypts its original gradients before sending to the smart contract. In detail, $P_{i}$ chooses random numbers $k_{P_{i}} \in \mathbb{Z}_{N}^{*}$, and executes the following operations to encrypt $\triangle \mathbf{W}_{t}^{i}$ with the public key $P K=N$.

$$
C\left(\Delta \mathbf{W}_{i}^{t}\right)=g^{\Delta \mathbf{W}_{i}^{t}} \cdot k_{P_{i}}^{N} \bmod N^{2}
$$

And $P_{i}$ provides a publicly auditable proof $\operatorname{Proof}_{P K_{t, i}}$ to guarantee encryption correctness.

$$
\operatorname{Proof}_{P K_{t, i}}=\text { fsprove }_{P K}\left(\Sigma_{P K} ; C\left(\triangle \mathbf{W}_{i}^{t}\right) ; \Delta \mathbf{W}_{i}^{t}, k_{P_{i}} ; p k_{P_{i}}\right)
$$

Step 4: Gradient Upload. Each party $P_{i}$ sends an gradient transaction $\operatorname{Tran}_{P_{i}}^{G r a d}$ to the smart contract, where

$$
\operatorname{Tran}_{P_{i}}^{\text {Grad }}=\left\{C\left(\triangle \mathbf{W}_{i}^{t}\right), \operatorname{Proof}_{P K_{t, i}}\right\}
$$

The smart contract verifies correctness of the encrypted gradients via function

$$
\operatorname{fsver}_{P K}\left(\Sigma_{p k}, C\left(\triangle \mathbf{W}_{i}^{t}\right) ; \operatorname{Proof}_{P K_{t, i}} ; p k_{P_{i}}\right)
$$

\subsection{Aggregation Phase}

Step 1: Model aggregation. Upon receiving the local updates from training devices before a preset time-stamp, each miner updates the global model according to the aggregating rule defined in the smart contract. Suppose that at the $t+1$-th iteration of collaborative training, local gradients $C\left(\Delta \mathbf{W}_{i}^{t}\right), i \in\{1, \ldots, n\}$, have been 
uploaded, then miners competitively execute update operations by

$$
C\left(\mathbf{W}^{t+1}\right)=C\left(\mathbf{W}^{t}\right) \cdot \frac{1}{n} \cdot \prod_{i=1}^{n} C\left(\Delta \mathbf{W}_{i}^{t}\right)
$$

Once update operation finished, miners then send the updated results $C\left(\mathbf{W}^{t+1}\right)$ through transactions to the smart contract.

Step 2: Block generation and propagation. Each miner starts mining until either it finds the nonce or it receives a generated block from other miners. The learning results are stored in the block as well. When one miner generates a new block, other miners verify the contents of this block (e.g., the nonce, the state changed by the smart contract, the transactions, and the aggregated model). If a block is verified by the majority of miners, this block will be added on the blockchain and accepted by the whole network.

Step 3: Reward allocation. The smart contract deployed by the task publisher rewards the miners as well as the training devices according to their contributions in the learning task.

\section{SECURITY ANALYSIS}

In this section, we analyze the security of the proposed PDFDL. Specifically, following the security requirements discussed earlier, our analysis focuses on how to preserve the private party's information during the federated deep learning process.

Theorem 1. PDFDL achieves the privacy of parties' local gradients $\Delta \mathbf{W}_{i}$ and the confidentiality of global model parameters $\mathbf{W}$ against honest-but-curious model. (i.e., miners want to obtain the underlying plaintext of $C\left(\Delta \mathbf{W}_{i}\right)$ and $\mathbf{W}$ for stealing devices' local gradients $\Delta \mathbf{W}_{i}$ and the global model parameters $\mathbf{W}$, meanwhile, parties expect to achieve each other's local gradients).

Proof. We illustrate that both $\triangle \mathbf{W}_{i}$ and $\mathbf{W}$ can be well protected during different phases of PDFDL.

- In the local gradients encryption phase, all elements are encrypted with the public key $P K=N$, and a local gradient $\Delta \mathbf{W}_{i}$ is transformed to $C\left(\Delta \mathbf{W}_{i}\right)$ by its owner via computing $\left[\left|p_{i j k}\right|\right]=g^{p_{i j k}} \cdot r_{i j k}^{N} \bmod N^{2}$, where $j=1, \cdots, t$ and $k=1, \cdots, m$. Note that $N$ is the public key of paillier cryptosystem with threshold decryption, and the corresponding secret key $S K$ is splitted into $n$ distributed secret keys $s k_{D_{1}}, \ldots, s k_{D_{n}}$ for parties. Since a sole distributed key $s k$ cannot retrieve the ciphertext $\left[\left|p_{i j k}\right|\right]$, therefore, it is impossible for a individual $D_{i}$ to decrypt the $C\left(\Delta \mathbf{W}_{i}\right)$. Moreover, since $m d p_{i j}$ is the sum of elements in vector $p_{i j k}$, miner cannot retrieve the elements of $p_{i j k}$ with $m d p_{i j}$. Thus, the privacy of $\Delta \mathbf{W}_{i}$ can be well protected in this phase.

- In the collaborative training phase, miner aggregates the encrypted gradients of $\left\{\Delta \mathbf{W}_{i}\right\}_{i \in[1, \cdots, n]}$ with the homomorphic characteristic of paillier crypto-system to obtain the global model parameters. In this process, only all parties partially decrypt the $a c p_{i^{\prime} j^{\prime} k}$, thus, it is impossible for the $E D^{\prime}$ to obtain the original data of $\Delta \mathbf{W}_{i}$.

In other words, $D_{i}$ can only obtain the global model parameters $C(\mathbf{W})_{i j}$, which is valueless for party but necessary for the entire process of collaborative model learning. Thus, during this phase, the privacy of $\left\{\Delta \mathbf{W}_{i}\right\}_{i \in[1, \cdots, n]}$ can be verified. In addition, due to all operations in miners are over ciphertext, miner finally obtain the encrypted global model parameters $C(\mathbf{W})$, which can only be decrypted by parties. Therefore, the confidentiality of $\mathbf{W}$ is guaranteed.

- In the collaborative learned result reading phase, all parties decrypt the $C(\mathbf{W})$ distributedly, and each $D_{i}$ shares its partially decryption result $C(\mathbf{W})_{D_{i}}$. Meanwhile, $C(\mathbf{W})$ can only be retrieved while the number of parties participating in the decryption is greater the threshold, thus, it is impossible for an individual $D_{i} \in \mathrm{D}$ to decrypt the final global model than other parties, which guarantees that all devices can obtain the final global model.

\section{PERFORMANCE EVALUATION}

\subsection{Evaluation Environment}

To measure overall performance, we perform PDFDL on the real federated data set FEMNIST [9]. The dataset contains 80, 5263 handwritten character images from 3550 different users and contains 62 different classes (10 digits, 26 lowercase, 26 uppercase). We simulated 900 parties in the training community, where the local datasets were not balanced in number and independent in distribution. We employed a private Ethereum blockchain named go-ethereum on an Intel Xeon CPU with a clock rate of $2.40 \mathrm{GHz}$ with 16 cores and 2 threads per core. The Smart Contract (SC) layer was developed using the Solidity programming language and deployed on the private blockchain using go-ethereum.

We build a deep learning environment using Python 3.7.6, numpy 1.14.0, and tensorflow 1.14.0 and deploy it on Geforce RTX 2080Ti GPUs. Meanwhile, we use Java to implement the Paillier cryptosystem, and set the number of bits of modulus $N$ to 1024, which is for a security level of 80 bits.

\subsection{Performance Analysis}

We compare our PDFDL with traditional federated deep learning (FDL) [13] and independent deep learning (DL) [6] as a baseline. Each shema classifies images using Convolution Neural Network $(\mathrm{CNN})$ model which has the following structure: Input $\rightarrow$ Conv $\rightarrow$ Maxpool $\rightarrow$ Fully Connected $\rightarrow$ Output. The weights and bias in Conv layer, Fully Connected layer and Output layer are set as $w_{1}=$ $(10,1,3,3)$ and $b_{1}=(10,1), w_{2}=(1960,128)$ and $b_{2}=(1,128), w_{3}=$ $(128,10)$ and $b_{3}=(1,10)$, respectively. In the BPFDL experiment, $k \%$ represents the proportion of parties in each training round. In FDL, $k \%$ also represents the proportion of training nodes. Meanwhile, stand-alone DL uses the entire dataset. Under the conditions of different $k$ values, we recorded their performance in Fig. 4.

As you can see from Figure 4, as the proportion of participants increases, the performance of PDFDL continues to approach the effect of FDL, with only a slight loss compared to a standalone DL with a complete data set. Compared with the standalone DL, PDFDL also has the privacy data protection function of federated learning, which does not require a trusted central server to manage, greatly reducing the risk of privacy leakage.

The malicious parties in the training community can produce harmful updates that, if integrated, can significantly degrade the performance of the global model. Malicious node attacks are performed to show how PDFDL and FDL are affected under different malicious proportions among parties. We assume that malicious actors are in collusion and that the attack mode of the malicious 


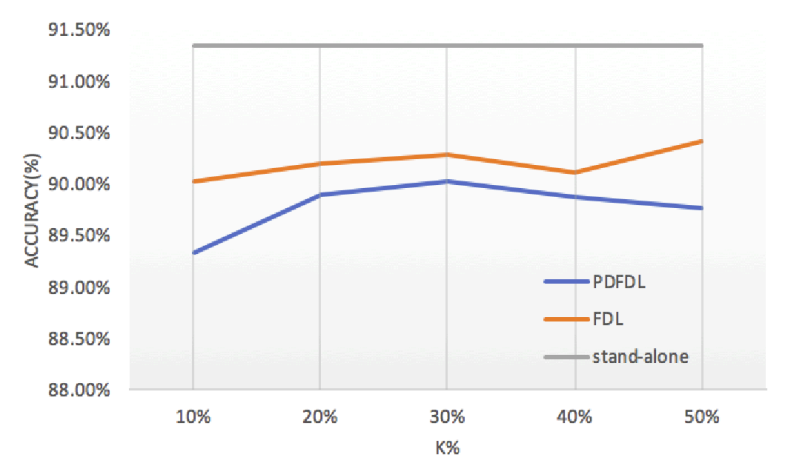

Figure 4: Accuracy of PDFDL, FDL and Stand-alone DL on FEMINST dataset with different proportion of active parties.

node is random perturbation with a point-wise Gaussian random noise. The FDL will not perform any defense measures, and model updates generated by randomly selected active parties will be integrated. BPFDL relies on blockchain based on multi-party secure computing technology mentioned above to resist the attack. And the proportion of parties is fixed as $20 \%$.

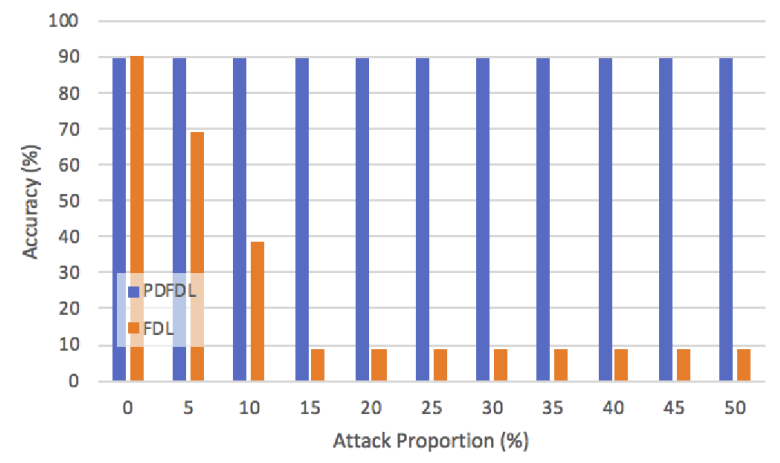

Figure 5: Performance of methods under malicious attacks.

As shown in Figure 5, the BPFDL can resist much higher malicious nodes proportion than FDL. This indicates the effectiveness of BPFDL with the help of blockchain based on multi-party secure computing technology.

\section{CONCLUSION}

In this paper, we proposed a novel privacy-preserving decentralized federated deep learning scheme, called PDFDL. Based on paillier cryptosystem with threshold decryption and secure multi-party aggregating computation, multiple parties can securely learn a more accurate global model with their private local training data in the assistance of blockchain, meanwhile, the confidentiality of the final global model can be ensured. Specifically, before being sent to the blockchain, all of the local model gradients are encrypted by their owner, and calculated without decryption during the decentralized federated deep learning process. Therefore, other parties cannot obtain each other's private training data, and miners cannot achieve any private information of parties, as well as the final global model. Detailed security analysis shows its security strength and privacypreserving ability, and extensive experiments were conducted to demonstrate its efficiency.

\section{ACKNOWLEDGMENTS}

This work was supported by Natural Science Foundation of Shaanxi (Grant No. 2013JM8022).

\section{REFERENCES}

[1] A. Bansal, T. Chen, and S. Zhong. 2011. Privacy preserving backpropagation neural network learning over arbitrarily partitioned data. Neural Computing Applications 20, 1 (2011), 143-150.

[2] T.H. Chan, K. Jia, S. Gao, J. Lu, Z. Zeng, and Y. Ma. 2015. Pcanet: A simple deep learning baseline for image classification? IEEE Transactions on Image Processing 24, 12 (2015), 5017-5032.

[3] P. Danaee, R. Ghaeini, and D. A. Hendrix. 2017. A deep learning approach for cancer detection and relevant gene identification. In PACIFIC SYMPOSIUM ON BIOCOMPUTING 2017, World Scientific (Ed.). 219-229.

[4] E. Gawehn, J. A. Hiss, and G. Schneider. 2016. Deep learning in drug discovery. Molecular informatics 35, 1 (2016), 3-14.

[5] István Hegedűs, Gábor Danner, and Márk Jelasity. 2019. Gossip Learning as a Decentralized Alternative to Federated Learning. In Distributed Applications and Interoperable Systems, José Pereira and Laura Ricci (Eds.). Springer International Publishing, Cham, 74-90.

[6] G. Hinton, L. Deng, D. Yu, G. E. Dahl, A. Mohamed, N. Jaitly, A. Senior, V. Vanhoucke, P. Nguyen, T. N. Sainath, and B. Kingsbury. 2012. Deep Neural Networks for Acoustic Modeling in Speech Recognition: The Shared Views of Four Research Groups. IEEE Signal Processing Magazine 29, 6 (2012), 82-97. https://doi.org/10.1109/MSP.2012.2205597

[7] B. Hitaj, G. Ateniese, and F. Perez-Cruz. 2017. Deep models under the gan: information leakage from collaborative deep learning. In Proceedings of the 2017 ACM SIGSAC Conference on Computer and Communications Security. ACM.

[8] Chenghao Hu, Jingyan Jiang, and Zhi Wang. 2019. Decentralized Federated Learning: A Segmented Gossip Approach.

[9] C. J. B. Yann LeCun and Corinna Cortes. 2021. The mnist database of handwritten digits. http://yann.lecun.com/exdb/mnist/

[10] Y. LeCun, Y. Bengio, and G. Hinton. 2015. Deep learning. nature 521, 7553 (2015), 436.

[11] T. Li, A. K. Sahu, A. Talwalkar, and V. Smith. 2020. Federated Learning: Challenges, Methods, and Future Directions. IEEE Signal Processing Magazine 37, 3 (2020), 50-60. https://doi.org/10.1109/MSP.2020.2975749

[12] Y. Liu, J. Peng, J. Kang, A. M. Iliyasu, D. Niyato, and A. A. A. El-Latif. 2020. A Secure Federated Learning Framework for $5 \mathrm{G}$ Networks. IEEE Wireless Communications 27, 4 (2020), 24-31. https://doi.org/10.1109/MWC.01.1900525

[13] H. McMahan, Eider Moore, Daniel Ramage, and Blaise Agüera y Arcas. 2016. Federated Learning of Deep Networks using Model Averaging. (02 2016).

[14] L. Melis, C. Song, E. D. Cristofaro, and V. Shmatikov. 2018. Inference attacks against collaborative learning. (2018). arXiv preprint arXiv:1805.04049.

[15] Paritosh Ramanan, Kiyoshi Nakayama, and Ratnesh Sharma. 2019. BAFFLE : Blockchain based Aggregator Free Federated Learning.

[16] R. Shokri and V. Shmatikov. 2015. Privacy-preserving deep learning. In 2015 53rd Annual Allerton Conference on Communication, Control, and Computing (Allerton). 909-910. https://doi.org/10.1109/ALLERTON.2015.7447103

[17] C. Song, T. Ristenpart, and V. Shmatikov. 2017. Machine learning models that remember too much. In Proceedings of the 2017 ACM SIGSAC Conference on Computer and Communications Security.

[18] J. Yuan and S. Yu. 2014. Privacy preserving back-propagation learning made practical with cloud computing. IEEE Transactions on Parallel Distributed Systems 25,1 (2014), 212-221.

[19] S. Zhou, H. Huang, W. Chen, P. Zhou, Z. Zheng, and S. Guo. 2020. PIRATE: A Blockchain-Based Secure Framework of Distributed Machine Learning in $5 \mathrm{G}$ Networks. IEEE Network 34, 6 (2020), 84-91. https://doi.org/10.1109/MNET.001. 1900658 